\title{
L'angle de Böhler dans le diagnostic de fracture du calcanéus
}

\section{Böhler's Angle for the Diagnosis of Calcaneus Fracture}

\author{
P. Kauffmann · F. Baicry $\cdot$ P. Le Borgne \\ (C) SFMU et Lavoisier SAS 2015
}

Une femme de 47 ans s'est présentée aux urgences pour une douleur du pied gauche suite à la chute d'un escabeau. L'examen clinique retrouve une impotence fonctionnelle complète avec une douleur diffuse de la cheville et de l'arrière-pied sans déformation ni hématome visible. La mobilité de la cheville est normale dans tous les plans mais limitée par la douleur. Le bilan radiographique initial est interprété comme normal (Fig. 1A). Le traitement proposé est symptomatique ; il ne permet qu'une amélioration partielle des symptômes. Un scanner du pied réalisé deux mois après le traumatisme retrouve une fracture du calcanéus avec enfoncement vertical du thalamus (Fig. 1B). L'angle de Böhler mesuré à $14^{\circ}$ sur la radiographie initiale aurait pu anticiper le diagnostic. La condensation osseuse au sein du calcanéus et l'encoche de son bord supérieur sont également des éléments évocateurs. L'angle décrit par Böhler (1931) se mesure sur une radiographie de profil du calcanéus en traçant deux lignes sur le relief supérieur de l'os : la première relie l'apex de la facette articulaire à l'apex du processus postérieur et la deuxième relie ce même apex articulaire à l'apex du processus antérieur. Les valeurs normales de cet angle oscillent entre $20^{\circ}$ et $40^{\circ}$ (Fig. 1C). Sa diminution signe l'abaissement de l'apex articulaire et le déplacement d'une probable fracture. L'importance du déplacement mesuré par cet angle est corrélée à la sévérité de la lésion [1]. L'apparition secondaire d'une ecchymose plantaire et/ou une douleur persistante à la palpation du calcanéus doivent faire évoquer ce diagnostic. Le traitement d'une fracture déplacée du calcanéus est habituellement chirurgical. En raison du délai de la prise en charge, cette patiente a bénéficié d'un traitement orthopédique (botte plâtrée sans appui pendant

P. Kauffmann · F. Baicry $\cdot$ P. Le Borgne $(\bowtie)$

Service d'accueil des urgences,

hôpitaux universitaires de Strasbourg, 1 avenue Molière,

F-67098 Strasbourg, France

e-mail : Pierrick_med@yahoo.fr

P. Le Borgne

Faculté de médecine, université de Strasbourg,

4 rue Kirschleger, F-67000 Strasbourg

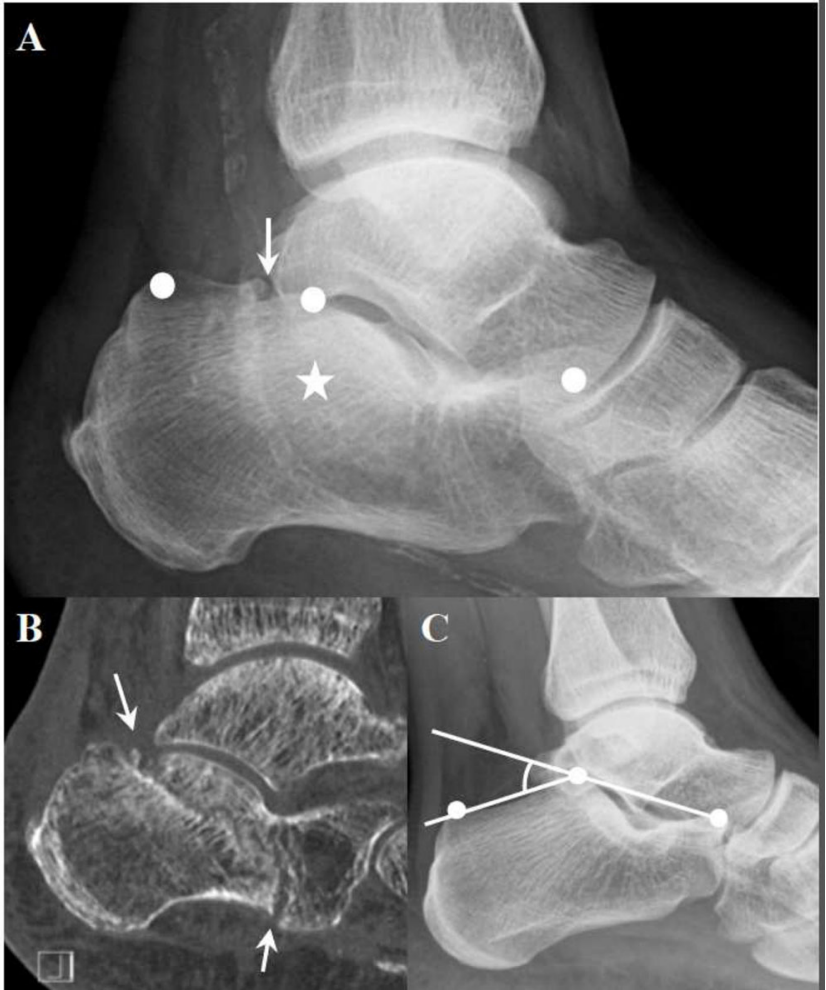

Fig. 1 A/ Radiographie standard pied gauche (profil). Points blancs : les trois apex du calcaneus permettant la mesure de l'angle de Böhler. Flèche: encoche du bord supérieur du calcanéus. Étoile : condensation osseuse. B/ Scanner pied gauche (coupe sagittale). Flèches : fracture du calcanéus avec enfoncement thalamique vertical. C/ Radiographie normale pied gauche (profil). Lignes : mesure de l'angle de Böhler

six semaines). À un an, la patiente conservait des douleurs du pied lors d'efforts soutenus.

\section{Références}

1. Su Y, Chen W, Zhang T, et al (2013) Böhler's angle's role in assessing the injury severity and functional outcome of internal fixation for displaced intra-articular calcaneal fractures: a retrospective study. BMC Surg 24:13-40 